\title{
Study on Web-Based Cotton Fertilization Recommendation and Information Management Decision Support System
}

\author{
Yv-mei Dang and Xin Lv* \\ Key Laboratory of Oasis Ecology Agriculture of Xinjiang Production and construction Group \\ Shihez, Xinjiang Province, P.R. China, 832003 \\ dym_glg@sina.com, lxshz@126.com
}

\begin{abstract}
Based on the regional difference in soil fertility, the nutrient uptake of crop, the nutrient supplying capacity of soil and the response of fertilizers, etc., this study took the 81th regiment of the 5th agricultural division in Xinjiang Bingtuan as the training and cooperative region, and established the information system of soil nutrients for cotton fields and recommendation model of fertilization. Constructed comprehensive, digital and intellectualized WEBbased cotton fertilization recommendation and information management decision support system by using SQL+JSP+Win2000.The system had been applied successfully in 2005 and 2006 there, and in 2006 the mean yield per $\mathrm{hm}^{2}$ increased by $7.3 \%$ than that of former 3 years, and the fertilizer cost per $\mathrm{hm}^{2}$ reduced 114 yuan(RMB).
\end{abstract}

Keywords: Knowledge model, Fertilization recommendation, Decision support system, SQL+JSP+Win2000.

\section{Introduction}

In this research ,it made collection and reorganization of field soil information from all regiments through network, provides the accurate and reliable soil material, take quantitative dynamic relationship as the master line that between the crops goal output, the soil nutrient conditions and fertilization measures, construct Dynamic Knowledge Model for cotton fertilization [1][2]; on this foundation, further unifies the soil fertility management system, constructs cotton information management system and Fertilization Decision Support System which are comprehensive, digitization and the intellectualization based on the platform of SQL+JSP+Win2000 with Web-based. the system has no high demand that only browser in clilnet, and provides a uniform interface, easy operation, easy Installation Maintenance[3].so as to provide the decision support for science fertilization more intuitively.

* Corresponding author.

D. Li, Y. Liu, and Y. Chen (Eds.): CCTA 2010, Part II, IFIP AICT 345, pp. 726-733, 2011.

(C) IFIP International Federation for Information Processing 2011 


\section{Material and Methods}

The system is composed of the fertilizer database ,cotton fertilizer and management system (knoweledge model and fertilizer model), the method storehouse, cotton fertilizer knowledge base, Inference Engine and technical personnel and man-machine connection.[3] (Fig.1)

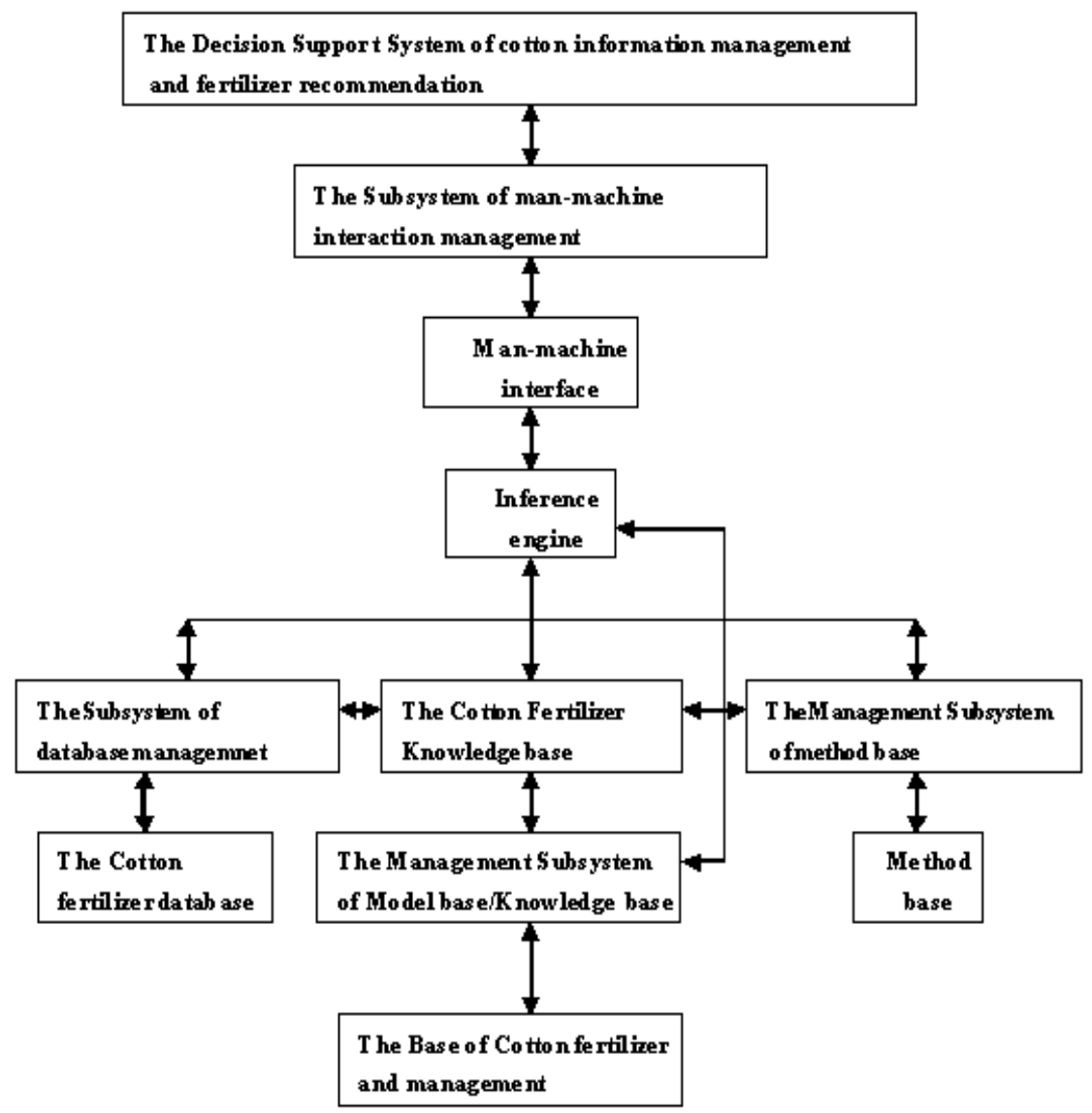

Fig. 1. Framework of system fuction

\subsection{Establishment of Foundation Database}

Establish cotton foundation databases by the information of 81th Regiment from 1998-2005 years about fields soil fertility, soil fertilizing kinds and quantity previous years, the variety and the output of crop planting, disease and insect pests and irrigation information and so on. The field soil fertilizer information be given by technician 
to take soil samples to make chemical test, the data be provided by Testing and Analysis Center of farm, to ensure the reliability and authenticity of information data.

\subsection{Establishment of Model and Model Base}

Based on test data, observation data and the agronomic data, we established the model of fertilizer recommendation, production prediction and Soil Evaluation, formed a model database of basic knowledge. It established the standardized fertilizer model for different soil fertility and yield level through experiment, accordance with all kinds of factors which established, combined with soil science, fertilizer science, as well as the local soil texture and expert experiences [4].Seeks the best economical fertilizer rate, the biggest profit fertilizer rate, the maximum yield fertilizer rate, best proportion and time distribution of fertilizer. Simultaneously, summarize the experience gradually and modify the original model, causes it to meet actual need. datas and the parameters can be called by each model, the result be returned to dynamic database, and realize resource sharing of database and model base.

\subsection{Establishment of System Knowledge Library}

There is main technical knowledge which are qualitative and cannot be expressed by model at present in cotton Cultivation Management knowledge system, involves prepares before sowing (seed treatment, ploughing, application method of base fertilizer, moisture management, forecasting and prevention of plants on pests and weed control), sowing date, density knowledge, the knowledge of crop nutrition and fertilizer, the knowledge of cotton moisture management, the knowledge of cotton growth and development, the knowledge of plant protection and so on. It including: variety selection knowledge base, density knowledge base, sowing date knowledge base, the relation between sowing date and accumulated temperature, suitable sowing date, the relation between cotton sowing date and yield formation, the knowledge base of fertilizer and crop nutrition, water management knowledge base, chemistry control knowledge base, pest and weed knowledge base and prevention knowledge base, plant protection.

\subsection{Method Base}

It is the tool to save the module of method, composed of various algorithm program which are more generality and flexibility.

\subsection{Inference Engine}

It is the logical core of the system make inference using the knowledge to data. It make inference to the data in database by controlling the knowledge base, and draws the new conclusion. In other words, the inference Engine is one kind of strategy procedure. Production inference strategy used in this system, the inference process is: 
$<$ Known > rule: If $\mathrm{A}$ then $\mathrm{B}$

$<$ Known > precondition: A< new > conclusion: B

\subsection{Connection of Man-Machine}

IE6 is basic interface in this system, can make interaction with user by draw menus, toolbars, icons, graphics and tables, selective prompting be given, you can make selection by menus, the whole operation can be completed only by computer mouse and keyboard, then user can get the model and output the results. Mean while, the system provides the help files to make explanation for using the system. In addition, the contact surface of system has the good fault tolerance too, gives the error message and the processing prompt through examination common mistake by setting error trap, to ensure the correctness by user input.

\section{Results and Discussion}

The system of cotton information management and cotton fertilization recommendation decision support system based on WEB realizes the main function such as :data management, system management, information query, fertilizer recommendation, soil evaluation, the expert knowledge and consultation, the result output and the system maintenance management and so on (Fig.2-5).

\subsection{Data Management Module}

There are 5 attribute database management module in the module[5] (soil basic information database, fertilizer information database, fertilizer amount of previous years, fertilizer parameter database, user information database), each administration module has the operation functions such as: increase, deletion, saves, printing, search, sorting, screening and so on. and give the right of remote input and revise to user, advanced user may renew the attribute data whenever, so make the database can always reflect the newest tendency of farmland nutrients and other management information. Also user may carry on maintenance to database and module base(mainly to data edition, update and so on).At first, data be inputed in the database, so as to be called for inquiry and recommendation.

\subsection{Information Inquiry Module}

Provides two ways to inquire for fertilizing scheme and field information. The user can copy the data from database according to the need, simultaneously the data in database can be batch introduced into through this contact surface. Information query and screening realizes information acquisiton function according to the query condition and screening condition the user combined to the data in database[5].The table for Inquiring or screening may be the table of expert system standard, also may be the result which the system recommendation decision-making leaves, the data message inquire may be printed directly and derive.(Fig.2) 


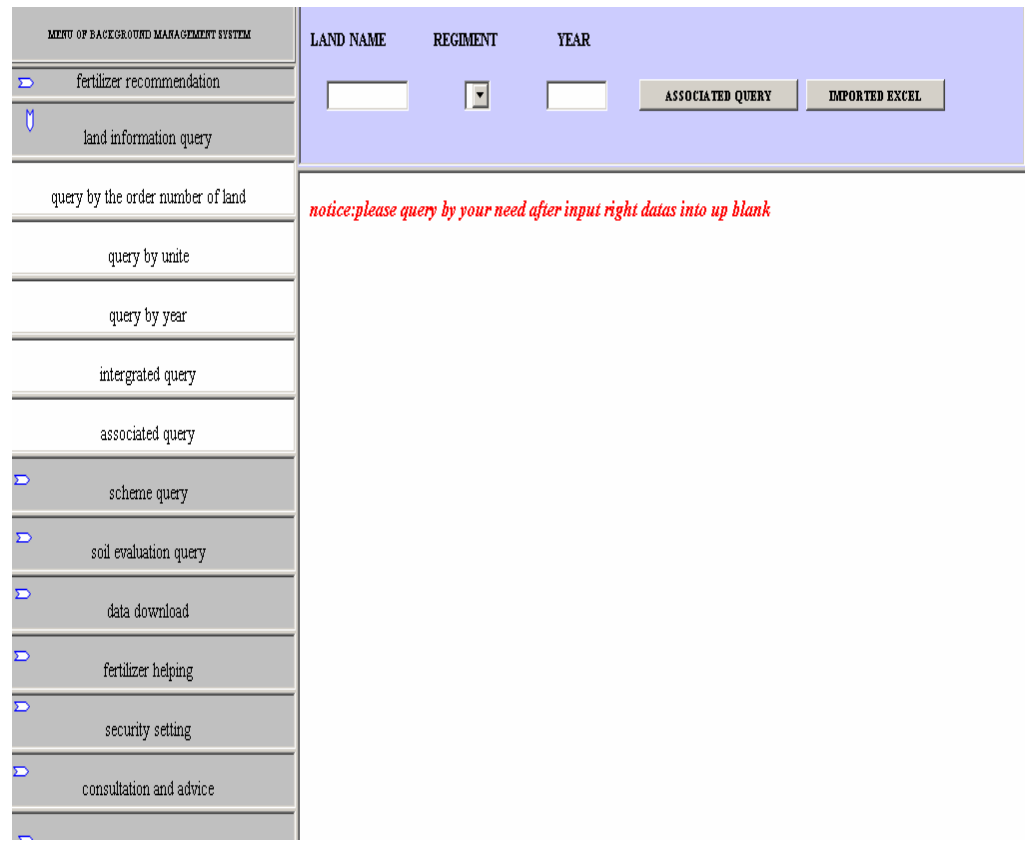

Fig. 2. Information Query

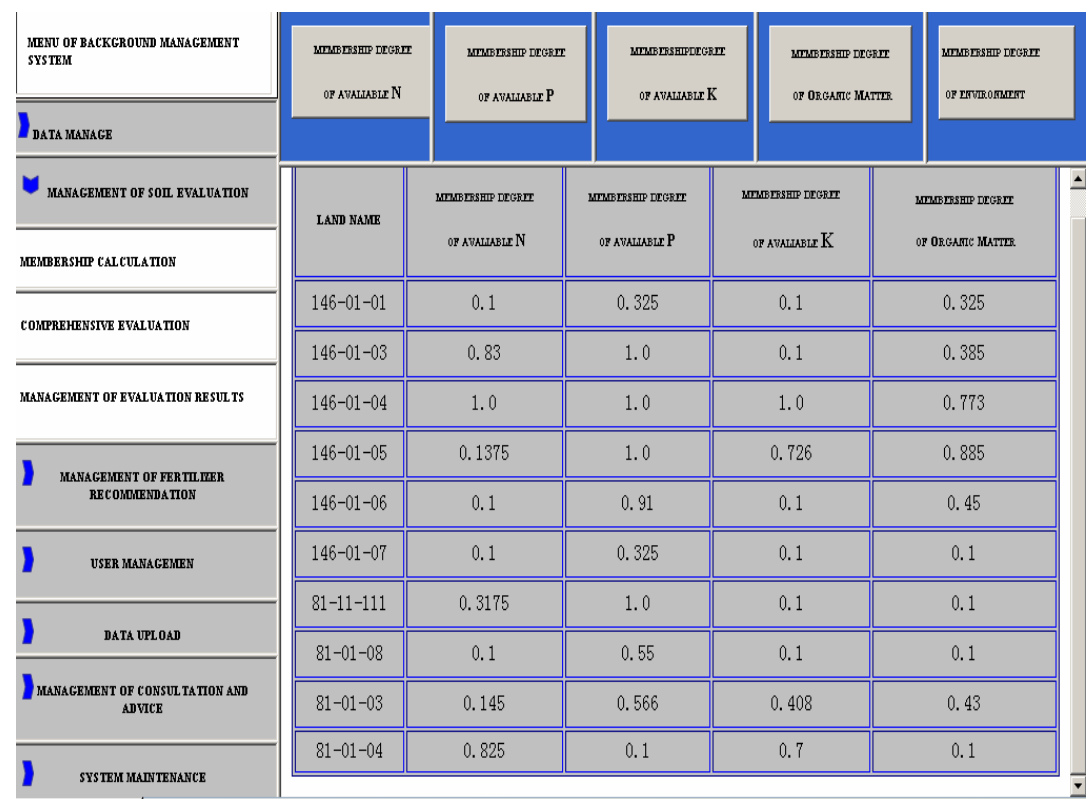

Fig. 3. Management of Soil evaluation 


\subsection{Module of Soil Appraisal}

Primary to compute membership degree of Organic Matter, available nitrogen, available $\mathrm{P}$, available N, and obtains the index value of comprehensive evaluation. (Fig.3)

\subsection{The Design of Soil Fertilizer Recommendation Module}

The module including conventional fertilization recommendation by soil test, drip irrigation fertilizer recommendation, effect function recommendation, organic fertilizer recommendation and microelements fertilizer recommendation. Under the suggestion of guide(Fig.4),the user can input the data of soil nutrient, fill in fertilizer amount of strip field, variety selection of fertilization and establish goal output according to local actual situation. The system judges whether the data user input is reasonable based on the ordinary years' data of meteorology, soil, variety and so through operating the knowledge model, if reasonable, then make fertilizer recommended by calling the knowledge module of fertilizer module, according to the information user filled in, the result of fertilizer recommendation can be printed on the formula to apply fertilizer card or data export by Excel, the user can modify, edits and prints the recommendation result by Excel datas exported. If unreasonable, the system modify the plan which have be made, and sends it to the fertilizer model to make forecast, so circulates, until generating a set of fertilizer recommendation plan which meet requirement[6].

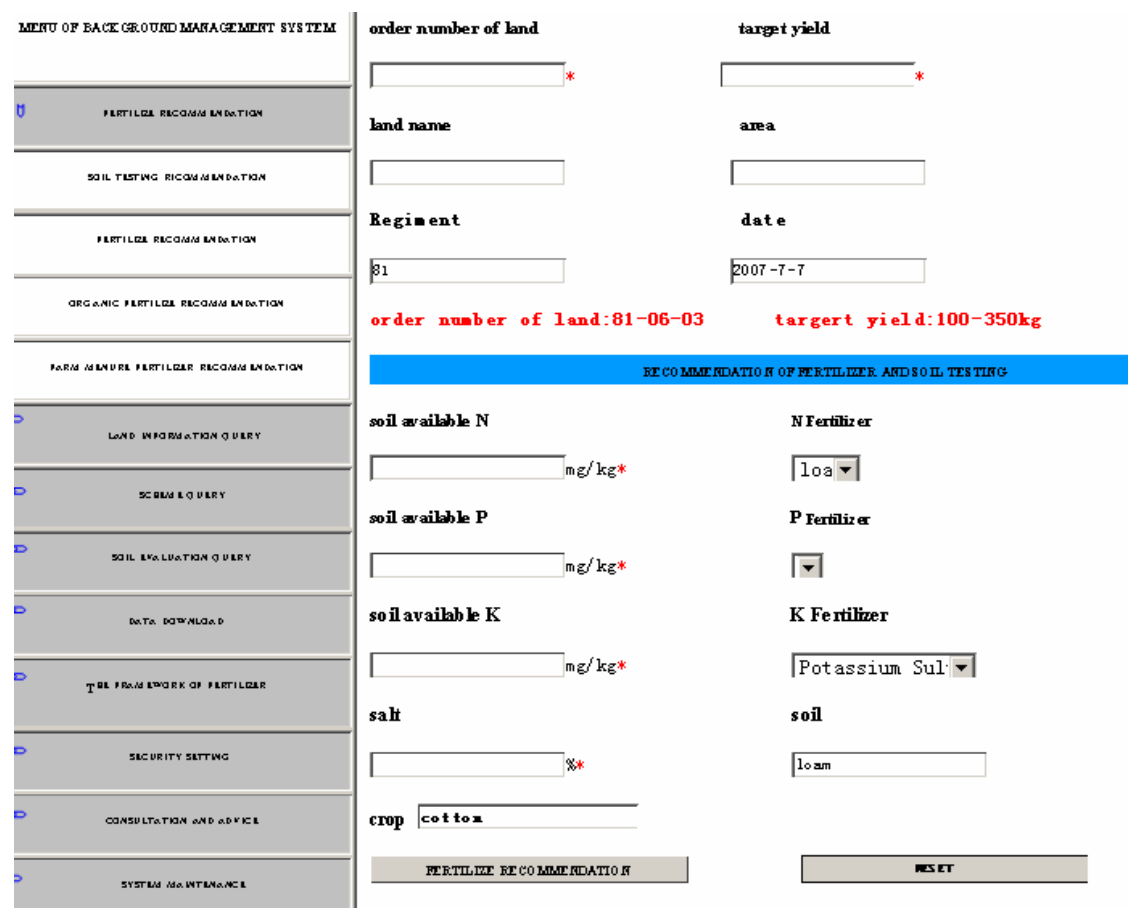

Fig. 4. Frame work of fertilize recommendation 


\subsection{Maintenance and Management of System}

The system gives different jurisdiction to the different grades user, the user may browse, inquire, modify, increase and delete the knowledge of the knowledge base and datas of database in own purview.( Fig.5)

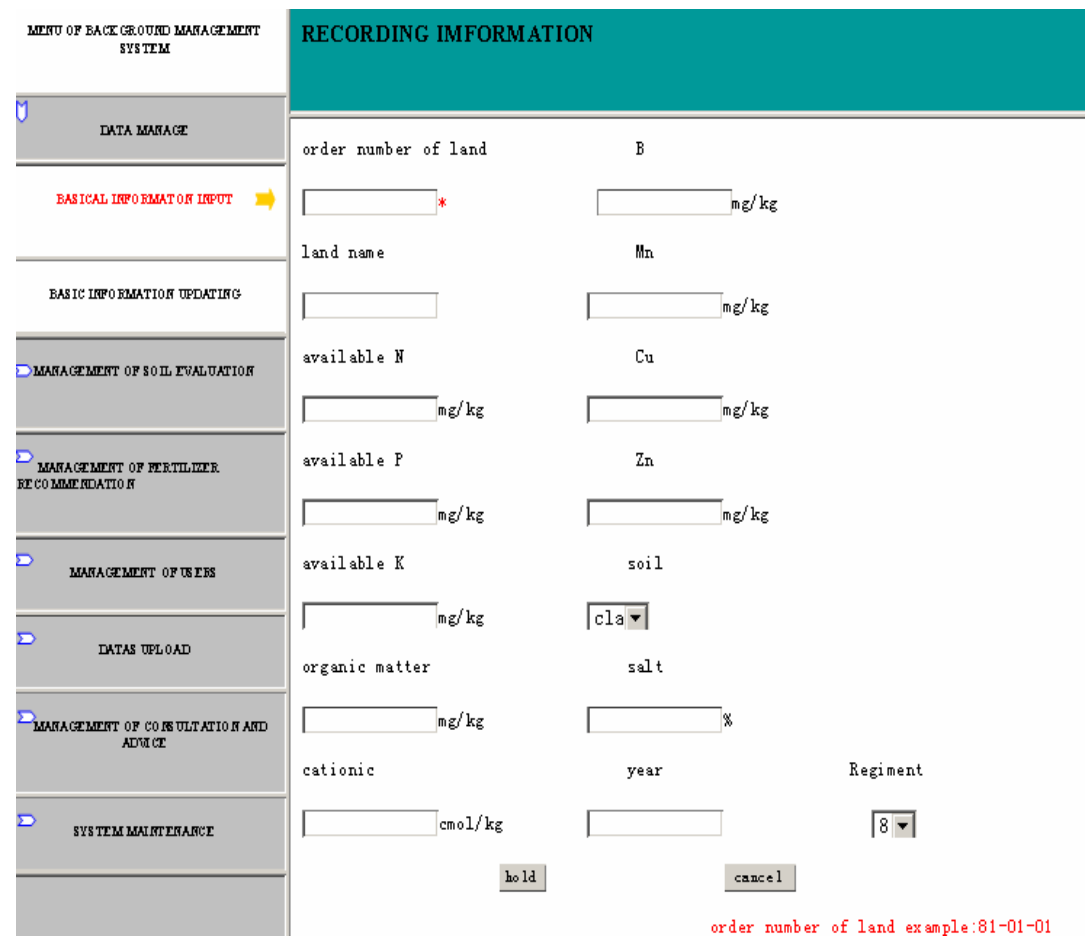

Fig. 5. Management and maintenance of database

\section{Conclusion}

Precise fertilization is important part of Precision Agriculture, It is the best fertilizer plan established above the scientific method to fertilizer.This system construct comprehensive digital and intelligent decision support system based on WEB using the SQL+JSP+Win2000, and collected and arrangemented soil information of all regiments by Internet, and provides the accurate and reliable soil material, then obtained the comprehensive index of soil fertility using fuzzy mathematics principle, make fertilizer recommendation, establish fertilization model of soil nutrient, the fertility district and the formula district of crop specific fertilizer; establish the balance fertilization system of nitrogen, the phosphorus, the potassium and the trace element according to the consideration about soil supplying nutrient capability 
and fertilizer needs of crops. It will be important theory value and the practice significance to implementing balance fertilization technique and generalization of other Scientific Research Achievements.

\section{References}

[1] Zhu, Y., Cao, W.x.: A Knowledge Model-and Growth Model-Based Decision Support System for Wheat Management. Sceintia Agricultura Sinica 37(6), 814-820 (2004)

[2] Hao, Y.-l., et al.: Programming Technology of J2EE. The Publishing House of Qinghua university and Beijing Jiaotong University, Beijing (2005)

[3] Xiong, F.1., Qiao, K.z., Hu, H.-y.: Agricultural Expert System and Development Tools. The Publishing House of Qinghua University, Beijing (1999)

[4] Yang, B.-r.: Knowledge Engineering and Knowledge Discovery, pp. 423-450. The Publishing House of Metallurgy Industry, Beijng (2000)

[5] Yan, C., Lv, X.: Information Management and Fertilizing Decision System Based on Soil Nutrient of SuperMap IS Cotton Field. XinJiang Agricultural Sciences 41(6), 427-430 (2004)

[6] Xie, K.-w., Cheng, Y.-q.: The Design and Implementation of the Expert System Based on Database. Journal of Hubei Institute For Nationalities (Natural science) (2), 193-196 (2005) 